\title{
Percepção de qualidade de vida de pessoas com fibrose cística: um estudo sobre a adequação dos instrumentos de medida
}

\author{
Perception of quality of life of persons with cystic fibrosis: a study on the adequacy \\ of rating scales
}

\author{
Juliana Bredemeier'1, William Barbosa Gomes² \\ ${ }^{1}$ Pós-graduanda em Psicologia do Desenvolvimento, Universidade Federal do Rio Grande do Sul (UFRGS), Porto Alegre, RS. ${ }^{2}$ Professor adjunto, \\ Curso de Pós-Graduação em Psicologia do Desenvolvimento, Instituto de Psicologia, UFRGS, Porto Alegre, RS.
}

\begin{abstract}
Resumo
Introdução: Avaliações de qualidade de vida (QV) na fibrose cística (FC) são tão importantes quanto a avaliação da condição clínica do paciente. Porém, há divergências sobre conceitos e teorias de QV.

Método: Onze pacientes brasileiros com FC maiores de 18 anos responderam a perguntas sugeridas pela OMS para a exploração conceitual da QV. A pesquisa foi orientada pela abordagem da grounded theory, e os dados sofreram análise qualitativa.

Resultados: Apesar da diversidade de domínios valorizados pelos entrevistados estar de acordo com o caráter multidimensional da QV, há domínios ainda não presentes nas escalas.

Conclusões: Nenhuma escala atualmente disponível abrange todos os aspectos valorados pelos pacientes, o que aponta para a importância de questões idiossincráticas e culturais para a QV na FC. O esclarecimento da relação entre saúde e QV ainda depende de pesquisas futuras.

Descritores: Escalas de avaliação, fibrose cística, qualidade de vida, qualidade de vida relacionada à saúde.
\end{abstract}

\begin{abstract}
Introduction: Quality of life (QOL) assessment in cystic fibrosis is as important as the assessment of the patient's health status. However, there are controversies on QOL concepts and theories.

Method: Eleven Brazilian patients with cystic fibrosis, 18 years or older, answered questions suggested by the World Health Organization to explore the concept of QOL. The grounded theory approach provided the basis for this study and the data were qualitatively analyzed.

Results: Although the diversity of domains valued by the participants is in agreement with the multidimensional nature of QOL, there are some domains missing in the scales.

Conclusions: No currently available scale comprises all aspects ranked as important by the patients. This underscores the importance of idiosyncratic and cultural aspects on QOL in cystic fibrosis. Clarifying the relationship between health and QOL still relies on further research.

Keywords: Rating scales, cystic fibrosis, quality of life, health-related quality of life.
\end{abstract}




\section{Introdução}

A fibrose cística (FC) é a doença crônica sistêmica de origem genética mais comum entre caucasóides, alcançando proporções de 1:2.500 nascidos vivos ${ }^{1}$. No Brasil, os números ainda não são definitivos, havendo registros de incidências no nascimento que variam de 1:1.850 até 1:9.600². Estima-se que aproximadamente 10 milhões de pessoas no mundo sejam portadoras assintomáticas da doença ${ }^{3}$.

Dentre as conseqüências mais graves da FC encontram-se insuficiência pancreática e deterioração progressiva da função pulmonar ${ }^{4}$. A doença apresenta uma grande variabilidade de sintomas, de gravidade e de progressão ${ }^{5}$. O tratamento é complexo, consome tempo, e deve ser realizado diariamente ${ }^{6}$.

Avanços ocorridos nas últimas décadas no tratamento da FC levaram a um aumento da sobrevida desses pacientes até a idade adulta ${ }^{2}$, o que gerou esforços para lhes propiciar o melhor bem-estar possível. Nesse sentido, avaliações de qualidade de vida (QV) têm se mostrado um importante subsídio para o desenvolvimento de intervenções médicas e psicológicas mais sensíveis e eficazes, levando em consideração a perspectiva do próprio paciente ${ }^{7}$. Avaliações de QV são importantes para o planejamento e acompanhamento do tratamento, para a identificação dos fatores preditores de QV e para o desenvolvimento de estratégias de promoção de bem-estar e de prevenção de saúde mental ${ }^{8-13}$.

Apesar de ainda não haver consenso sobre a definição de $\mathrm{QV}^{14-17}$, se aceita que subjetividade, multidimensionalidade e sentimentos positivos e negativos constituem elementos básicos do construto ${ }^{18-}$ 20. Para o Grupo de Estudos em Qualidade de Vida da OMS (Grupo WHOQOL) ${ }^{20}$, o conceito é definido como “a percepção do indivíduo de sua posição na vida no contexto da cultura e sistema de valores nos quais ele vive e em relação aos seus objetivos, expectativas, padrões e preocupações”21.

O desenvolvimento de instrumentos de medição da QV específicos para a FC foi embasado no fato de que instrumentos genéricos e instrumentos relacionados à saúde não se mostraram capazes de indicar variações na QV em função da gravidade da doença ${ }^{8,10,11}$. Com isso, foram desenvolvidos instrumentos para acessar os aspectos particularmente afetados pela ocorrência de $\mathrm{FC}^{10,19,22}$.

Contudo, a constatação de que instrumentos genéricos de medida de $\mathrm{QV}$ não são sensíveis a variações de gravidade da FC pode sugerir que a gravidade da doença talvez não seja o único e nem o mais importante determinante da QV. Há pesquisas que revelam que nem sempre as pessoas mais bem sucedidas - e nem tampouco as mais saudáveis - são as que alcançam maior pontuação em $\mathrm{QV}^{19}$. Nesse sentido, argumenta-se que instrumentos desenvolvidos com sensibilidade para esta variação de gravidade podem estar calibrados para medir outra coisa que não a QV ${ }^{15,16}$. Um estudo de Bowling ${ }^{18}$ sobre os aspectos considerados importantes pelas pessoas para a sua QV reforça essa possibilidade. A análise dos domínios incluídos nas escalas mais populares de medição de qualidade de vida relacionada à saúde (QVRS) revelou que certos domínios apontados como importantes pelos participantes não eram contemplados pelos instrumentos.

Com isso, há na atualidade um grande cuidado na

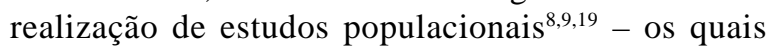
envolvem o levantamento de experiências, de informações, de sentimentos e de crenças da população alvo sobre QV - antes de se desenvolver um instrumento de medida. Uma vez desenvolvido, o instrumento pode ser traduzido e validado para novos idiomas e culturas. Porém, a validação de uma escala levanta um novo problema - o da transculturalidade do conceito. Sabese que o processo de tradução e de validação não garante que o construto pelo qual se tem interesse faça sentido na nova cultura ${ }^{23,24}$. Além disso, mesmo que investigações com o instrumento não apresentem evidências para um viés cultural, isso não significa que não existam diferenças que discriminem os grupos significa apenas que as diferenças culturais examinadas não afetam significativamente as propriedades psicométricas do instrumento ${ }^{25}$.

Na FC, até onde sabemos, dispõem-se atualmente de três escalas já publicadas para a medição da QV relacionada à doença, todas desenvolvidas a partir da revisão de literatura e da investigação da opinião de peritos e de pacientes. No Brasil, recentemente, foram publicados $^{26}$ os resultados iniciais dos processos de tradução e validação do Cystic Fibrosis Questionnaire - Revised (CFQ-R) ${ }^{27}$. Entretanto, praticamente não há relatos de estudos sobre QV na FC em nosso país.

Considerada a importância da avaliação de QV na população de pacientes com FC, três justificativas principais embasam a realização deste estudo: 1) a ausência de estudos exploratórios sobre o tema da QV com pacientes com FC no Brasil; 2) a importância da clarificação do conceito QV na população investigada, prévia ou simultaneamente ao desenvolvimento de instrumentos de medida; e 3) a importância da consideração da percepção do próprio respondente sobre a avaliação de sua QV.

O objetivo principal do presente estudo foi explorar e compreender a perspectiva de pacientes com FC sobre os aspectos determinantes de sua QV. Os objetivos complementares foram: 1) compreender qualitativamente como os pacientes de FC percebem as relações entre doença e QV; e 2) definir domínios 
importantes para avaliações de QV na FC a partir da percepção dos pacientes. Este estudo foi parte de uma investigação maior que buscou explorar a experiência de crescer e desenvolver-se com FC e os aspectos importantes para a QV destes pacientes.

\section{Método}

\section{Participantes}

Foram entrevistados 11 pacientes brasileiros maiores de 18 anos, recrutados através de duas associações de amparo a portadores da mucoviscidose no estado do Rio Grande do Sul (RS). O tamanho da amostra do estudo geral foi determinado segundo critérios de saturação e redundância ${ }^{28}$. Dos 13 pacientes contatados por telefone, um não concordou em participar e um não compareceu à entrevista. Não foi obtida resposta dos dois pacientes convidados por carta, computando-se assim um índice de participação de 73,33\%. Características sociodemográficas e dados referentes ao diagnóstico dos participantes podem ser vistos na Tabela 1 . Todos os entrevistados eram atendidos em um mesmo centro de referência para FC no RS. As entrevistas foram realizadas entre agosto de 2004 e fevereiro de 2005.

Tabela 1 - Descrição das características dos participantes

\begin{tabular}{lc}
\hline $\mathrm{n}$ & 11 \\
Mulheres & 7 \\
$\begin{array}{l}\text { Idade mediana (mín. e máx.) } \\
\begin{array}{l}\text { Idade mediana no diagnóstico } \\
\text { (mín. e máx.) }\end{array}\end{array}$ & $23,6(18,5-46,7)$ \\
$\begin{array}{l}\text { Mediana de anos de escolaridade } \\
\text { (mín. e máx.) }\end{array}$ & $11,8(5-40)$ \\
$\begin{array}{l}\text { Mediana de anos de conhecimento } \\
\text { do diagnóstico (mín. e máx.) }\end{array}$ & $12,13(0,5-27,6)$ \\
\hline
\end{tabular}

\section{Instrumentos}

\section{Roteiro de entrevista}

Envolveu quatro perguntas recomendadas pela Organização Mundial da Saúde (OMS) ${ }^{29}$ para a exploração da QV (Tabela 2).

Tabela 2 - Resumo das respostas conferidas às questões gerais sobre qualidade de vida

\begin{tabular}{|c|c|c|c|}
\hline $\begin{array}{l}\text { Definição de qualidade de } \\
\text { vida }\end{array}$ & $\begin{array}{l}\text { O que é importante para } \\
\text { qualidade de vida }\end{array}$ & $\begin{array}{l}\text { O que prejudica seu bem- } \\
\text { estar }\end{array}$ & $\begin{array}{l}\text { O que melhoraria sua } \\
\text { qualidade de vida }\end{array}$ \\
\hline $\begin{array}{l}\text { Sentir-se bem } \\
\text { Bem-estar } \\
\text { Felicidade } \\
\text { Ter boas condições de vida }\end{array}$ & $\begin{array}{l}\text { Ter saúde } \\
\text { Praticar exercícios físicos } \\
\text { Alimentar-se bem } \\
\text { Realizar o tratamento } \\
\text { Enfrentar a doença } \\
\text { Não se estressar } \\
\text { Otimismo } \\
\text { Saber o que fazer para se } \\
\text { sentir bem } \\
\text { Força de vontade } \\
\text { Trabalho, ocupação } \\
\text { Família, amigos, } \\
\text { relacionamento amoroso } \\
\text { Receber cuidados, ajuda e } \\
\text { atenção } \\
\text { Morar bem, ter conforto } \\
\text { Dinheiro } \\
\text { Estudo } \\
\text { Acesso a serviço de } \\
\text { atendimento de saúde de } \\
\text { qualidade } \\
\text { Acesso a medicações }\end{array}$ & $\begin{array}{l}\text { Falta de ar, tosse } \\
\text { Ser tratado como } \\
\text { "coitadinho", sentir-se } \\
\text { incapaz } \\
\text { O tempo gasto com o } \\
\text { tratamento } \\
\text { Não conseguir um trabalho } \\
\text { em função do tempo gasto } \\
\text { com o tratamento } \\
\text { A vergonha com o } \\
\text { tratamento } \\
\text { Pouca oferta de centros de } \\
\text { tratamento } \\
\text { Demora no diagnóstico } \\
\text { Falta de perspectiva de } \\
\text { futuro } \\
\text { Poluição, trocas de } \\
\text { temperatura }\end{array}$ & $\begin{array}{l}\text { Não ter FC, um transplante } \\
\text { pulmonar } \\
\text { Nada pode melhorar, porque } \\
\text { já está tudo muito bom } \\
\text { Nada pode melhorar, porque } \\
\text { não há o que fazer (tudo está } \\
\text { ruim) } \\
\text { Um corpo mais "sarado" } \\
\text { Não ter a fisioterapia ou a } \\
\text { saúde como uma } \\
\text { preocupação diária } \\
\text { Não depender do tratamento } \\
\text { para poder trabalhar com o } \\
\text { que desejasse } \\
\text { Conversas com outros } \\
\text { pacientes para troca de } \\
\text { experiências } \\
\text { Ter ajuda com as tarefas do } \\
\text { tratamento } \\
\text { Residir em local de clima } \\
\text { mais ameno }\end{array}$ \\
\hline
\end{tabular}




\section{Questionário}

Roteiro breve com perguntas sociodemográficas e clínicas desenvolvido com base nos itens sociodemográficos do CFQ-R ${ }^{27}$.

\section{Procedimentos}

Para controlar uma possível influência de uma condição clínica desfavorável na percepção de QV, nenhum dos participantes foi entrevistado durante internação hospitalar. Os residentes na capital foram entrevistados em suas casas; os de outras cidades, no consultório da pesquisadora em data de consulta de rotina. Todos assinaram termo de consentimento livre e esclarecido, no qual foi garantida a desistência a qualquer momento do estudo.

Os entrevistados foram estimulados a responder de acordo com suas experiências, informações, sentimentos e opiniões pessoais. As entrevistas foram realizadas sempre pela mesma entrevistadora (JB) e duraram, no total, entre 45-90 minutos, sendo gravadas e, posteriormente, transcritas na íntegra. Os entrevistados receberam garantia de confidencialidade, e a pesquisa foi aprovada pelo Comitê de Ética da Universidade Federal do Rio Grande do Sul (Processo número 2005380).

\section{Análise}

A análise das entrevistas seguiu os pressupostos da grounded theory $(\mathrm{GT})^{30}$. Essa abordagem foi escolhida por ser um método muito útil em assuntos sobre os quais existe pouca ou nenhuma pesquisa, pois permite a identificação de variáveis dentro de problemas comportamentais complexos ${ }^{31}$. A GT é considerada uma teoria emergente, um método de análise e um guia para o pesquisador analisar seus dados.

Segundo a GT, a coleta de dados e a análise não são apenas simultâneas, mas também circulares, o que permite mudanças de foco à medida que os dados sugerirem essa necessidade. É essa circularidade também chamada de método comparativo constante que permite a eficiência do critério de saturação, que se refere ao processo de sinalização de que dados suficientes já foram coletados ${ }^{32}$. Já a suspensão de idéias a priori, outro recurso da GT, é o que permite a sensibilidade teórica para depreender a perspectiva das entrevistas e confere maior validade aos achados ${ }^{30,32}$.

Neste estudo, a análise pressupôs (1) a leitura exaustiva de todas as entrevistas, o que permitiu a demarcação de categorias a partir da perspectiva dos entrevistados; (2) a leitura e a exploração dessas categorias para a definição de enunciados que sintetizaram as descrições iniciais; e (3) a comparação das unidades temáticas levantadas nas descrições com os itens presentes em escalas de avaliação de QV. A revisão da literatura perpassou todas as etapas da análise, refletindo novamente a circularidade do método. Exatamente por isso os achados serão apresentados em conjunto com a discussão elaborada a partir dos mesmos.

\section{Resultados}

A noção de QV oferecida pelos pacientes está de acordo com o caráter multidimensional do construto (Tabela 2). A exploração do conceito a partir das perguntas embasadas nas sugestões da OMS revelou a associação de QV com termos como "sentir-se bem”, "bem-estar" e "felicidade", como já evidenciado na literatura ${ }^{29}$. A saúde foi um dos aspectos considerados importantes para se ter uma boa QV, mas a avaliação da própria QV dos entrevistados não incluiu necessariamente a descrição de sintomas. As efetivas referências a sintomas envolveram primordialmente limitações decorrentes de problemas pulmonares: "não respiro bem”, “canso até pra subir uma escada”, ou "meu pulmão já não é mais o mesmo”.

Dentre os aspectos não mencionados destaca-se a ausência de referências à espiritualidade. A espiritualidade é associada a bem-estar físico e mental, sendo atualmente considerada um domínio tão importante na determinação da QV quanto os domínios físico e psicológico ${ }^{33}$. Salienta-se que a falta de menção dos pacientes a questões espirituais não significa que a espiritualidade não seja um domínio importante. Outros aspectos de igual importância podem também não ter sido mencionados.

A percepção de QV apresentou-se marcada pelas singularidades de cada entrevistado. Há quem acredite que para se ter QV é necessário “não ter fibrose cística”. Ao mesmo tempo, há quem diga ter uma boa QV apesar da FC, podendo viver melhor do que quem não possui uma doença crônica: “(...) eu vivi [até hoje] muito melhor que minha mãe, $e$, no entanto, tenho fibrose”.

Em função das demandas da doença, é possível que o paciente considere ter tido melhor QV na infância, quando infecções e limitações provocadas pela FC têm ocorrência mais baixa: "era mais feliz quando não tinha preocupação com saúde”. Em contrapartida, pacientes diagnosticados na adultez reconhecem que a possibilidade de início precoce do tratamento, bem como os benefícios dele decorrentes, poderiam ter proporcionado uma melhor QV na infância.

O tratamento que o paciente deve realizar tem relação paradoxal com a QV. Por um lado, pode ser percebido como prejudicial à $\mathrm{QV}$ em função do tempo e da dedicação demandados. Por outro, pode ser percebido como algo que proporciona desfrutar uma 
vida boa: "No caso a demora, né, o tempo que a gente perde, né [fazendo o tratamento]... perde entre aspas, né, porque não é um tempo perdido, né, é um tempo ganho, estou ganhando qualidade de vida”. A importância de realizar o tratamento adequado foi reconhecida, mas alertando que algumas vezes o acesso ao tratamento não é garantia de que ele será realizado.

O apoio recebido do(a) companheiro(a), de familiares, amigos, professores e colegas de aula e de trabalho surgiu como um aspecto que influencia a QV. Quando o diagnóstico foi realizado na adultez, o companheiro foi o primeiro a receber a notícia e a oferecer apoio. A união familiar apareceu como algo que auxilia o enfrentamento da doença, tanto pelo afeto quanto pelo auxílio oferecido pelos familiares na realização do tratamento. A oportunidade de conversar com outros pacientes com FC - como ocorre em grupos de sala de espera - e os atendimentos oferecidos por profissionais da área da saúde mental foram também mencionados como fatores de apoio.

$\mathrm{O}$ modo como os pacientes enfrentam a doença revela-se na percepção expressa da QV. Os participantes que consideraram que a FC é uma barreira para a realização de atividades foram também aqueles que se auto-avaliaram com uma QV ruim. Complementarmente, aqueles que mencionaram que a realização do tratamento proporciona bem-estar e que é possível ser feliz apesar da presença de uma doença se auto-avaliaram com boa QV.

\section{Análise dos instrumentos de medida de QV na FC}

Cinco instrumentos de medição de QV foram analisados: um instrumento genérico (WHOQOL100) ${ }^{34}$, um relacionado à saúde (Short Form-36) ${ }^{35}$ e três instrumentos relacionados à FC: 1) Cystic Fibrosis Questionnaire - Revised ${ }^{27}$, versão norte-americana do Cystic Fibrosis Questionnaire (CFQ) $)^{22}$, originalmente publicado em francês; 2) Cystic Fibrosis Quality of Life (CFQoL) ${ }^{9}$; e 3) Questions on Life Satisfaction - CF Module $\left(\mathrm{FLZ}^{\mathrm{M}}-\mathrm{CF}\right)^{19}$. O FLZ ${ }^{\mathrm{M}}$-CF pressupõe o uso conjunto de um módulo genérico (FLZ ${ }^{\mathrm{M}}$ General) e de um módulo relacionado à saúde (FLZ ${ }^{\mathrm{M}}$ Health). Essa análise é apresentada na Tabela 3.

Tabela 3 - Aspectos importantes para a qualidade de vida, na perspectiva dos pacientes entrevistados, em comparação com os domínios presentes em uma escala genérica (WHOQOL-100), uma relacionada à saúde (SF-36) e em três escalas relacionadas à doença, utilizadas na medição da QV na FC

\begin{tabular}{|c|c|c|c|c|c|}
\hline & $\begin{array}{l}\text { WHOQOL-100 } \\
\text { (100 itens) }\end{array}$ & $\begin{array}{c}\text { SF-36 } \\
(36 \text { itens })\end{array}$ & $\begin{array}{c}\text { CFQ-R } \\
\text { (50 itens) }\end{array}$ & $\begin{array}{c}\text { CFQoL } \\
\text { (52 itens) }\end{array}$ & $\begin{array}{c}\text { FLZ }^{\mathrm{M}} \text { General, }^{\mathrm{FLZ}} \mathbf{Z}^{\mathrm{M}} \\
\text { Health, FLZ }{ }^{\mathrm{M}}-\mathbf{C F} \\
\text { (total: } 50 \text { itens) }\end{array}$ \\
\hline Funcionamento físico & Sim & Sim & Sim & Sim & Sim \\
\hline $\begin{array}{l}\text { Cuidados com a saúde } \\
\text { (alimentação, exercícios físicos) }\end{array}$ & Sim & Sim & Sim & Sim & Sim \\
\hline Auto-estima & Sim & Sim & Sim & Sim & - \\
\hline Sentimentos positivos & Sim & Sim & - & - & - \\
\hline Sentimentos negativos & Sim & Sim & Sim & Sim & Sim \\
\hline Imagem corporal & Sim & - & Sim & Sim & - \\
\hline Trabalho/ocupação & Sim & Sim & Sim & Sim & Sim \\
\hline Dependência do tratamento & Sim & Sim & Sim & Sim & Sim \\
\hline Adesão ao tratamento & - & - & Sim & - & Sim \\
\hline Independência & Sim & - & - & Sim & Sim \\
\hline Limitações causadas pela doença & Sim & Sim & Sim & Sim & Sim \\
\hline Relações sociais & Sim & Sim & Sim & Sim & Sim \\
\hline Apoio social & Sim & - & - & - & Sim \\
\hline Moradia & Sim & - & - & - & Sim \\
\hline Estudo & Sim & - & Sim & Sim & - \\
\hline Recursos financeiros & Sim & - & - & - & Sim \\
\hline $\begin{array}{l}\text { Acesso a serviço de saúde e a } \\
\text { medicamentos }\end{array}$ & Sim & - & - & - & - \\
\hline Poluição e clima & Sim & - & - & - & - \\
\hline Perspectiva de futuro & - & - & Sim & Sim & - \\
\hline
\end{tabular}


Apenas os instrumentos WHOQOL-100 e SF36 apresentam versões já publicadas em português brasileiro $^{34,36}$. Por isso, os três instrumentos relacionados à doença foram analisados a partir de suas versões em inglês.

Na Tabela 3, verifica-se que os itens "acesso a serviço de saúde e a medicamentos" e "poluição e clima” não estão presentes em nenhum dos três instrumentos específicos para a FC. Esse achado salienta a possibilidade de existência de diferenças importantes na definição de QV na perspectiva de pacientes com FC no Brasil, em comparação aos países onde os instrumentos foram originados. Essas diferenças são provavelmente decorrentes de singularidades culturais. Na verdade, a importância de aspectos como "acesso a serviços assistenciais" e "meio-ambiente" para a saúde já foi registrada inclusive na legislação brasileira, que também aponta como determinantes e condicionantes básicos da saúde a alimentação, o saneamento básico, a educação, o transporte, o lazer, a renda, o acesso a bens, o trabalho e a moradia, entre outros ${ }^{37}$.

Os itens "recursos financeiros" e "moradia” são avaliados apenas no módulo de satisfação geral com a vida do FLZ ${ }^{\mathrm{M}}$. "Estudo" é avaliado no CFQ-R, no CFQoL e no WHOQOL-100. A menção espontânea feita pelos entrevistados a esses aspectos é facilmente explicada ao atentar-se para as condições socioeconômicas do Brasil, que em muito diferem da situação da Europa ou dos EUA, locais de desenvolvimento dos instrumentos. Esses cinco itens ("acesso a serviço de saúde e a medicamentos", "poluição e clima", "recursos financeiros", "moradia" e "estudo"), também não medidos pelo instrumento relacionado à doença (SF-36), aparecem nas facetas do WHOQOL-100.

A análise das formulações das perguntas e itens dos cinco questionários mostrou que o CFQ-R, o CFQoL e o SF-36 baseiam a mensuração da QV, em grande parte, na avaliação da extensão em que os aspectos funcionais do paciente são limitados pela doença. Já o WHOQOL-100 e o FLZ ${ }^{\mathrm{M}}$ buscam avaliações que levam em consideração a importância que o respondente confere a cada domínio, o que está em acordo com a perspectiva subjetiva do construto QV. O FLZ ${ }^{\mathrm{M}}$ realiza isso apresentando uma seção em que o respondente deve atribuir a importância de cada item na determinação de sua QV. Por exemplo, ao invés de perguntar quanto tempo o respondente ocupa com o tratamento, ou com que freqüência ele se levantou nas últimas duas semanas por causa da tosse (itens 16 e 46 do CFQ-R), pergunta-se quão importante é para a pessoa desempenhar o tratamento de forma consistente e quão importante é para ela estar livre de tosse (itens A6 e 1 do $\mathrm{FLZ}^{\mathrm{M}}$-CF)*. De maneira semelhante ao $\mathrm{FLZ}^{\mathrm{M}}-\mathrm{CF}$, o WHOQOL-100 apresenta diversos itens voltados à obtenção dessa avaliação de importância.

A comparação dos itens levantados nas entrevistas com os itens presentes nos instrumentos analisados revelou que o WHOQOL-100 é a escala que mais contempla os aspectos afetados pelas condições socioeconômicas brasileiras, presentes no Domínio V (Ambiente). Note-se que WHOQOL-100 foi desenvolvido com base na metodologia WHOQOL, a qual pressupõe a realização simultânea de grupos focais com indivíduos de diferentes idiomas e culturas, prévia e concomitantemente ao desenvolvimento das escalas $^{20,34}$. Sobre isso, há diversos autores que argumentam que o processo de tradução e de validação de uma escala nem sempre preserva a sensibilidade para características idiossincráticas da nova cultura e no novo idioma $^{23-25,38-41}$.

Frisby ${ }^{30}$ ressalta ainda que mesmo que investigações com o novo instrumento não levantem evidências de um viés cultural, isso pode dever-se ao fato de que as diferenças culturais examinadas podem não afetar significativamente as propriedades psicométricas do instrumento. Dizendo isso, o autor menciona o perigo já salientado por autores ${ }^{23-24}$ de que interpretações e conclusões errôneas sejam fornecidas a partir da pressuposição de que os testes original e adaptado são equivalentes.

Todas essas preocupações sobre a aplicabilidade de instrumentos adaptados levaram a International Test Comission (ITC) a desenvolver um conjunto de etapas para garantir a validade de construto e a fidedignidade do teste adaptado ${ }^{42}$. Entre outros aspectos, essas etapas enfatizam a necessidade de se avaliar grupos diferentes antes de presumir a existência de equivalência do construto entre as culturas. Assim, entendemos que apenas a exploração prévia do conceito, bem como a identificação de peculiaridades culturais e idiomáticas, é capaz de revelar se a estratégia mais acertada para permitir a avaliação compreensiva do construto, na nova população, seria a tradução e a validação do instrumento original ou o desenvolvimento de um novo instrumento.

A análise lingüística dos cinco instrumentos revelou ainda importantes diferenças na enunciação das perguntas e na elaboração dos itens. É possível

\footnotetext{
* CFQ-R 14+, item 16: "How much time do you currently spend each day on your treatments?”

CFQ-R 14+ item 46: "How often during the past two weeks have you woken up during the night because you were coughing?”

FLZM-CF, item A6: "How important is it for you to carry out daily therapy consistently?”

FLZM-CF, item 1: "How important is it for you to be free of breathing difficulties / coughing?”
} 
que essas diferenças possam gerar vieses importantes na avaliação da QV. Afinal, se alguém investe regularmente três horas de seu dia para fazer o tratamento, sem considerar isso importante para sua $\mathrm{QV}$, perguntas como "Quantas horas do seu dia você dispensa para realizar o tratamento?” estarão medindo outro aspecto desse indivíduo, que não sua QV. Isso indica ser necessário cautela sobre as conclusões tomadas a partir da constatação de que instrumentos genéricos não são sensíveis a variações da gravidade da FC. É possível que os instrumentos julgados como sensíveis estejam calibrados para essa variação justamente por perguntarem quantas vezes, na última semana, o respondente teve que acordar no meio da noite por causa de sua tosse (item 46, CFQ-R), ao invés do quão importante é para esse respondente estar livre de dificuldades respiratórias e tosse (item 1, FLZ ${ }^{\mathrm{M}}$ CF).

Quanto ao caráter determinante da saúde na QV, os três instrumentos relacionados à FC aqui analisados avaliam a QVRS, que já foi definida como não dependente de questões como renda e estilo de moradia $^{16}$. Em contraste, as entrevistas deste estudo revelaram que moradia, dinheiro, ambiente e acesso a atendimento de saúde e medicamentos são aspectos considerados importantes pelos pacientes. Isso sugere que a avaliação da QV na FC talvez não possa ser realizada apenas em termos de aspectos relacionados à doença.

\section{Discussão}

A exploração do relato das entrevistas revelou que aspectos como (1) apoio social, (2) sentimentos positivos e negativos, (3) estratégias de coping, (4) perspectiva de futuro e (5) momento do diagnóstico têm relação com a QV na FC. Os quatro primeiros aspectos já foram apontados por outras pesquisas como importantes para a QV em outras patologias ${ }^{43}$. Apesar disso, parece não haver pesquisas que relacionem esses aspectos e a QV na FC. Quanto ao momento do diagnóstico, também não temos conhecimento de nenhuma pesquisa até o presente momento que investigue a relação desse aspecto com a QV em qualquer população.

Outro achado relevante foi a revelação de que, de acordo com a perspectiva dos pacientes, a QV não está necessariamente relacionada à presença da doença. Já era sabido que nem sempre as pessoas mais saudáveis são as que apresentam melhor $\mathrm{QV}^{19}$. Conforme os dados das entrevistas, há pessoas com FC que acreditam ter vivido até hoje uma vida muito melhor do que outras pessoas sem a doença, o que indica que a FC nem sempre determina a satisfação de uma pessoa com sua vida. Além disso, verificamos ser possível que pacientes que se consideram com uma condição clínica ruim avaliem sua QV como boa ou muito boa. Nesse sentido, pesquisas vêm apontando que a depressão pode ter um papel mediador na avaliação dos pacientes sobre sua QV, o que talvez explique essas diferenças perceptuais ${ }^{44}$. Porém, ainda assim não podemos declarar que a QV na FC não está relacionada à presença da doença, pois há pacientes para quem o bem-estar parece ser diretamente dependente da saúde e dos aspectos funcionais limitados pela doença.

A análise das cinco escalas revelou que nenhuma delas abrange todos os aspectos valorados pelos pacientes como importantes para sua QV. OWHOQOL100, de desenvolvimento simultâneo e multicêntrico, foi a escala que mais abrangeu os domínios levantados a partir das entrevistas dos participantes deste estudo. Porém, esse instrumento tem como limitação não medir o peso do prejuízo funcional especificamente causado pela FC. Em contrapartida, os quatro instrumentos restantes falham em avaliar aspectos mais ambientais da vida dos respondentes. Apenas o WHOQOL-100 e o FLZ $^{\mathrm{M}}$ contemplam a importância da valorização individual do respondente a cada domínio mensurado.

Isto posto, é de nossa opinião que a máxima médica “cada caso é um caso” é de boa aplicação na definição subjetiva de QV. No Brasil, o acesso a serviços de saúde e a medicamentos pode ser difícil e eventualmente impraticável para famílias de baixa renda. Os relatos de nossos entrevistados apontaram que a peculiaridade da realidade socioeconômica influencia a experiência de ter FC, tornando-a diferente da experiência de uma pessoa nascida em um país desenvolvido. Por isso, consideramos que questões culturais, socioeconômicas e individuais que repercutem na QV - tal como o acesso a medicações, presente no WHOQOL-100, mas ausente em todos os instrumentos relacionados à FC - devem ser explorados, identificados e incluídos nas escalas de medida.

\section{Considerações finais}

Como implicação para a prática clínica, nossos dados apontam para a importância de não se perder o sentido da individualização do atendimento médico em seus desdobramentos biomédicos e psicossociais, o que já foi salientado por autores como Engel ${ }^{45}$ décadas atrás. As diferentes perspectivas individuais encontradas com relação à QV apontam a necessidade de se ultrapassar a noção de oferta de saúde básica para a noção de atenção integral à saúde, enfocando a importância do atendimento multiprofissional e individualizado na saúde. Sugerimos também a realização de investigações 
sobre personalidade e percepção de QV, cuja interação já foi apontada pela literatura ${ }^{46}$ e enfatizamos a importância de investigações que cruzem dados objetivos de status clínico - provas de função pulmonar e índices de massa corporal, por exemplo - com dados subjetivos na investigação da QV na FC.

Como base em nossas conclusões sobre a análise dos instrumentos, fazemos as seguintes sugestões para a pesquisa e uso de instrumentos de medida de QV:

1) que a exploração do conceito de QV com pessoas com FC, assim como a consulta a peritos e à literatura local, façam parte integrante do processo de decisão entre a tradução e a validação de um instrumento já existente ou o desenvolvimento de uma nova escala;

2) que a escolha pela tradução e validação seja feita apenas quando investigações exploratórias apontarem no sentido de uma equivalência do conceito nas culturas original e alvo;

3) que seja dada preferência à utilização de escalas que levam em consideração a importância do caráter subjetivo da QV, contemplando esse aspecto na escolha de itens e perguntas e no estilo lingüístico utilizado;

4) que a medição da QV na FC, dada a falta de consenso verificada inclusive entre os entrevistados deste estudo sobre o papel da saúde na determinação da QV, seja realizada com clareza conceitual e teórica sobre os aspectos determinantes da QV para a população alvo, e que o modelo teórico de base de cada instrumento utilizado seja sempre registrado nos relatos das investigações;

5) que investigações futuras se esforcem para estabelecer se o conceito de QVRS trata-se de um construto com efeito na vida dos pacientes, ou de uma definição operacional utilizada para explicar o enfoque nos aspectos sintomáticos e funcionais dos instrumentos de QV desenvolvidos a partir desta perspectiva; e

6) que a avaliação da QV seja encarada como um processo diagnóstico, em que etapas como entrevista e aconselhamento tenham tanta importância quanto a aplicação de um instrumento de medida.

Este estudo utilizou uma abordagem qualitativa de investigação, o que não permite a generalização de seus resultados para outras populações ou culturas. Os dados foram obtidos a partir de uma amostra particular selecionada por conveniência e atendida num mesmo local. Ainda assim, os achados desta investigação devem ser considerados com a mesma importância que é eventualmente conferida aos resultados derivados de pesquisas realizadas com quantidades expressivas de participantes. Segundo Brandão ${ }^{47}$, a fala de um indivíduo é na verdade um recorte das representações de um tempo histórico e de um espaço social. Assim, falas como "vivo muito bem apesar da doença” não necessariamente se referem apenas a histórias individuais, podendo refletir a história de diversos outros pacientes afetados pela FC.

Finalizamos dizendo que as opiniões aqui registradas podem refletir a opinião de muitos outros pacientes; podem ser transferidas ou extrapoladas para outras realidades e merecem atenção em investigações futuras.

\section{Referências}

1. Adde FV, Abreu e Silva FA, Reis FJC, Britto MCA, Damaceno N, Ludwig Neto N, et al. Roteiro para diagnóstico da fibrose cística. São Paulo: Laboratórios Roche; 2002.

2. Raskin S. Como a genética pode contribuir para o diagnóstico e tratamento da fibrose cística. In: Workshop de Fibrose Cística, $1^{\circ}$ Simpósio Nacional de Genética Clínica e Pediatria, XVII Congresso Brasileiro de Genética Clínica; 2005 Jun; Curitiba, Brasil.

3. Cystic Fibrosis Foundation (CFF). Patient registry: 2000 Annual Data Report. Maryland: CFF; 2001.

4. Scattolin I, Beier S, Blacher J, Laurent MC, Santos MIS. Desenvolvimento da atenção integral à criança com fibrose cística. In: Carvalho PRA, Ceccim RB, eds. Criança hospitalizada: atenção integral como escuta à vida. Porto Alegre: UFRGS; 1997. p. 90-5.

5. Abreu e Silva FA, Palombini BC. Fibrose cística: mucoviscidose. In: Silva LCC, org. Compêndio de pneumologia. São Paulo: Fundação Byk; 1991. p. 977-85.

6. Quittner AL, Opipari LC, Regoli MJ, Jacobsen J, Eigen H. The impact of caregiving and role strain on family life: comparisons between mothers of children with cystic fibrosis and matched controls. Rehabil Psychol. 1992;37:275-90.

7. Berlim MT, Fleck MPA. "Qualidade de vida”: um novo conceito para a pesquisa e prática em psiquiatria. Rev Bras Psiquiatr. 2003;25(4):249-52.

8. Henry B, Aussage P, Grosskopf C, Goehrs JM. Development of the Cystic Fibrosis Questionnaire (CFQ) for assessing quality of life in pediatric and adult patients. Qual Life Res. 2003;12(1):63-76.

9. Gee L, Abbott J, Conway SP, Etherington C, Webb AK. Development of a disease specific health related quality of life measure for adults and adolescents with cystic fibrosis. Thorax. 2000;55(11):946-54.

10. Quittner AL. Measurement of quality of life in cystic fibrosis. Curr Opin Pulm Med. 1998;4(6):326-31.

11. Quittner AL. Qualidade de vida na fibrose cística. In: Workshop em fibrose cística. Roche; 2003 Jul; São Paulo, Brasil.

12. Rozov T. Qualidade de vida em fibrose cística. In: Workshop de fibrose cística, $1^{\circ}$ Simpósio Nacional de Genética Clínica e Pediatria, XVII Congresso Brasileiro de Genética Clínica; 2005 Jun; Curitiba, Brasil.

13. Minayo MCS, Hartz ZMA, Buss PM. Qualidade de vida: um debate necessário. Ciênc Saúde Coletiva. 2000;5(1):7-18.

14. Doward LC, McKenna SP. Evolution of quality of life assessment. In: Rajagopalan R, Sheretz EF, Anderson RT, eds. Care management of skin diseases: life quality and economic impact. New York: Marcel Dekker; 1997. p. 9-33.

15. Hunt SM. The problem of quality of life. Qual Life Res. 1997;6(3):205-12.

16. van Heck GL. O conceito de qualidade de vida. In: II Encontro IberoAmericano de Qualidade de Vida; 2004 Ago; Porto Alegre, Brasil.

17. Ware J. A avaliação do SF-36 e o porvir: a próxima geração de medidas. In: II Encontro Ibero-Americano de Qualidade de Vida; 2004 Ago; Porto Alegre, Brasil.

18. Bowling A. What things are important in people's lives? A survey of the public's judgements to inform scales of health related quality of life. Soc Sci Med. 1995;41(10):1447-62.

19. Goldbeck L, Schmitz TG, Henrich G, Herschbach P. Questions on life satisfaction for adolescents and adults with cystic fibrosis: development of a disease-specific questionnaire. Chest. 2003;123(1):42-8.

20. The World Health Organization quality of life assessment 
(WHOQOL): position paper from the World Health Organization. Soc Sci Med. 1995;41(10):1403-9.

21. Fleck MPA. O instrumento de avaliação de qualidade de vida da Organização Mundial da Saúde (WHOQOL-100): características e perspectivas. Ciênc Saúde Coletiva. 2000;5(1):33-8.

22. Henry B, Grosskopf C, Aussage P, Goehrs JM, Launois R, French CFQoL Study Group. Construction of a disease-specific quality of life questionnaire for cystic fibrosis. Pediatr Pulmonol. 2003;13(Suppl):337-8.

23. van der Vijver F, Hambleton RK. Translating tests: some practical guidelines. Europ Psychol. 1996;1(2):89-99.

24. Hambleton RK, Patsula L. Adapting tests for use in multiple languages and cultures. Soc Indic Res. 1998;45:153-71.

25. Frisby CL. Culture and cultural differences. In: Sandoval J, Frisby CL, Geisinger KF, Scheuneman JD, Grenier JR, eds. Test interpretation and diversity. Washington, DC: American Psychological Association; 1998. p. 51-73.

26. Rozov T, Cunha MT, Nascimento O, Quittner AL, Jardim JR. Linguistic validation of cystic fibrosis quality of life questionnaires. $\mathrm{J}$ Pediatr (Rio J). 2006;82(2):151-6.

27. Quittner AL, Modi AC, Watrous M, Davis MA. The Cystic Fibrosis Questionnaire (CFQ): user's manual. Washington, DC: Cystic Fibrosis Foundation; 2000.

28. Lincoln Y, Guba E. Naturalistic inquiry. New York: Sage; 1985.

29. Fleck MPA, Chachamovich E, Trentini C. WHOQOL-OLD project: method and focus group results in Brazil. Rev Saude Publica. 2003;37(6):793-9

30. Glaser B, Strauss A. Discovery of grounded theory: strategies for qualitative research. New York: Aldine; 1967.

31. Stern PN. Grounded theory methodology: its uses and processes. Image (IN). 1980;12(1):20-3.

32. Hutchinson S. Grounded theory: The method. In: Munhall L, Boyd C, eds. Nursing research: a qualitative perspective. 2nd ed. New York: National League for Nursing; 1993. p. 180-212.

33. Fleck MPA, Borges ZN, Bolognesi G, Rocha NS. Desenvolvimento do WHOQOL módulo espiritualidade, religiosidade e crenças pessoais. Rev Saude Publica. 2003;37(4):446-55.

34. Organização Mundial da Saúde, Divisão de Saúde Mental, Grupo WHOQOL. Versão em português dos instrumentos de avaliação de qualidade de vida (WHOQOL). 1998. Disponível em: http:// www.ufrgs.br/psiq/whoqol1.html.

35. Ware JE Jr., Sherbourne CD. The MOS 36-item short-form health Survey (SF-36). I. Conceptual framework and item selection. Med Care. 1992;30(6):473-83.

36. Ciconelli RM. Tradução para o português e validação do questionário genérico de avaliação de qualidade de vida "Medical Outcomes Study 36-Item Short Form Health Survey” (SF-36) [tese]. São Paulo: Universidade Federal de São Paulo; 1997.

37. Brasil, Ministério da Saúde. Lei $N^{\circ} 8.080$, de 19 de Setembro de 1990. Disponível em: http://conselho.saude.gov.br/legislacao/ lei8080_190990.htm.

38. Geisinger KF. Cross-cultural normative assessment: translation and adaptation issues influencing the normative interpretation of assessment instruments. Psychol Assess. 1994;6(4):304-12.

39. Geisinger KF. Psychometric issues in test interpretation. In: Sandoval J, Frisby CL, Geisinger KF, Scheuneman JD, Grenier JR, eds. Test interpretation and diversity. Washington, DC: American Psychological Association; 1998. p. 17-30.

40. Helms JE. Why is there no study of cultural equivalence in standardized cognitive ability testing? Am Psychol. 1992;47(9):1083-101.

41. Hui CH, Triandis HC. Measurement in cross cultural psychology. J Cross Cult Psychol. 1985;16(2):131-52.

42. ITC, International Test Comission. Test Adaptation Guidelines: April 21, 2000 Version; 2000. Disponível em: www.intestcom.org/ test_adaptation.htm\#Context.

43. Remor E. Apoyo social y calidad de vida en la infección por el VIH. Aten Primaria. 2002;30(3):143-9.

44. Fleck MPA. Depresión y calidad de vida en enfermedades crônicas. In: $11^{\circ}$ Congreso Internacional de Medicina Interna del Hospital de Clínicas; 2006 Ago. Buenos Aires, Argentina. Palestra apresentada no Simposio "Calidad de vida en la práctica clínica".

45. Engel GL. The need for a new medical model: a challenge for biomedicine. Science. 1977;196(4286):129-36.

46. Chou CY, Brauer DJ. Temperament and satisfaction with health status among persons with rheumatoid arthritis. Clin Nurse Spec. 2005;19(2):94-100.

47. Brandão HHN. Introdução à análise do discurso. 2. ed. Campinas: Unicamp; 1993. 